\title{
Review Article \\ Review of Physical Based Monitoring Techniques for Condition Assessment of Corrosion in Reinforced Concrete
}

\author{
Ying Lei ${ }^{1}$ and Zhu-Peng Zheng ${ }^{1,2}$ \\ ${ }^{1}$ Department of Civil Engineering, Xiamen University, Xiamen 361005, China \\ ${ }^{2}$ Department of Mechanical \& Electrical Engineering, Xiamen University, Xiamen 361005, China
}

Correspondence should be addressed to Ying Lei; ylei@xmu.edu.cn

Received 30 October 2013; Revised 6 December 2013; Accepted 7 December 2013

Academic Editor: Ting-Hua Yi

Copyright ( 2013 Y. Lei and Z.-P. Zheng. This is an open access article distributed under the Creative Commons Attribution License, which permits unrestricted use, distribution, and reproduction in any medium, provided the original work is properly cited.

\begin{abstract}
Monitoring the condition of steel corrosion in reinforced concrete (RC) is imperative for structural durability. In the past decades, many electrochemistry based techniques have been developed for monitoring steel corrosion. However, these electrochemistry techniques can only assess steel corrosion through monitoring the surrounding concrete medium. As alternative tools, some physical based techniques have been proposed for accurate condition assessment of steel corrosion through direct measurements on embedded steels. In this paper, some physical based monitoring techniques developed in the last decade for condition assessment of steel corrosion in RC are reviewed. In particular, techniques based on ultrasonic guided wave (UGW) and Fiber Bragg grating (FBG) are emphasized. UGW based technique is first reviewed, including important characters of UGW, corrosion monitoring mechanism and feature extraction, monitoring corrosion induced deboning, pitting, interface roughness, and influence factors. Subsequently, FBG for monitoring corrosion in RC is reviewed. The studies and application of the FBG based corrosion sensor developed by the authors are presented. Other physical techniques for monitoring corrosion in RC are also introduced. Finally, the challenges and future trends in the development of physical based monitoring techniques for condition assessment of steel corrosion in RC are put forward.
\end{abstract}

\section{Introduction}

Reinforced concrete (RC) makes up a large part of the civil infrastructures. For instance, over half of the building and bridge inventories in China are reinforced concrete. Normally, concrete reinforced with steel is a rather splendid combination in which concrete also provides a high alkaline environment for the steel, helping to protect it from corrosion. However, corrosion of steel is inevitable, especially when RC structures are exposed to harsh environments with high carbon dioxide or chloride concentrations. The resulting corrosion products occupy volumes $6-10$ times that of the steel [1]. The increased volume induces tensile stresses in the concrete which result in cracking, delamination, and spalling. As a result, the reinforcements get exposed to direct environmental attack and the corrosion is accelerated. Corrosion, if undiscovered can cause catastrophic failure of the structure, is currently one of the primary durability concerns for RC structures. Corrosion causes billions of US dollars to be spent annually in repair, rehabilitation, and reconstruction efforts of RC structures, which makes it arguably the single largest infrastructural problem facing the industrialized countries [2]. It is imperative to develop effective monitoring techniques for condition assessment of corrosion in reinforced concrete.

A wide range of techniques have been reported in the literature that may be suitably employed for the monitoring of corrosion of steel in concrete structures for the purpose of diagnosing the cause and extent of the reinforcement corrosion [1-3]. Most of the existing techniques are electrochemical techniques such as half cell potential mapping and linear polarization which relate corrosion rate and extent through assessment on surrounding concrete medium. While many electrochemical techniques have been well-established, none of these techniques concentrate on corrosion monitoring through direct condition assessment or measurements 
on embedded steel. As alternative tools for monitoring steel corrosion, some physical based techniques have been proposed $[4,5]$. Compared with the electrochemistry based approaches, these physical approaches can not only provide supplemented tools for monitoring steel corrosion, but also conduct more accurate condition assessment of steel corrosion. However, there are still many challenges and difficulties of these physical based monitoring techniques for practical application of condition assessment of corrosion in reinforced concrete because the reinforcements are typically embedded inside concrete. Therefore, it is still imperative to investigate effective monitoring techniques for early detection of corrosion in steel embedded in concrete. Among the proposed physical based monitoring techniques, techniques based on ultrasonic guided wave (UGW) and fiber Bragg grating (FBG) have many advantages for monitoring corrosion-related damage in reinforcing bars, so they gained popularity among researchers in the past decades. This paper reviews some physical based monitoring techniques with emphases on the UGW and FBG based monitoring techniques for the condition assessment of steel corrosion in reinforced concrete in the last decade. The challenges and future trends in the development of physical based monitoring techniques for condition assessment of corrosion in reinforced concrete are also put forward. The structure of the rest of the paper is as follows. Section 2 reviews the UGW based techniques for corrosion monitoring of RC, which contains some important characters of UGW, mechanism of monitoring corrosion and feature extraction based on UGW, monitoring corrosion induced deboning, pitting, and interface roughness, respectively, and monitoring combined effects in actual corrosion and so forth; Section 3 reviews FBG for monitoring corrosion in RC, which contains mechanism of the FBG based corrosion sensor, design of FBG corrosion sensor proposed by the authors, and application of the designed FBG corrosion sensors in Xiamen Xiangan Subsea Tunnel in China; Section 4 introduces some of other physical techniques for monitoring corrosion in RC. Finally, the challenges and future trends in the development of physical based monitoring techniques for condition assessment of corrosion in reinforced concrete are summarized in the conclusions.

\section{UGW Based Techniques for Corrosion Monitoring of RC}

2.1. Some Important Characters of Guided Waves. In an infinite isotropic solid medium only two types of independent wave propagation exist, that is, compression and shear waves. When geometry constraints are introduced and the dimensions are close to the wavelength, the wave becomes dispersive and is called a guided wave. The propagation of guided waves is in accordance with the elastic theory in elastic media, whereas they follow the viscoelastic theory in nonelastic media. Pipes, anchor molts, rails, and steel rebar are typical waveguides.

There are three types of propagating waves in a cylindrical waveguide: longitudinal, torsional, and flexural waves due to complex effect of boundaries and they have frequencydependent properties with notations of $L(m, n), T(m, n)$, and
$F(m, n)$, respectively [6]. The characters " $m$ " and " $n$ " represent the circumferential displacement and the sequential order of the mode, respectively.

The displacement is symmetrical corresponding to $m=0$, where as $m=1,2,3, \ldots$ refers to asymmetrical displacements. Longitudinal modes that propagate in the steel rebar only have two types of displacement, namely, radical displacement $\mu_{r}$ and axial displacement $\mu_{z}$. Considering the boundary condition $\sigma_{r r}=\sigma_{r z}=0(r=a)$, the Pochhammer frequency equation can be achieved by solving the following wave equation [6]:

$$
\begin{aligned}
& \frac{2 \alpha}{a}\left(\beta^{2}+k^{2}\right) J_{1}(\alpha a) J_{1}(\beta a)-\left(\beta^{2}-k^{2}\right) J_{0}(\alpha a) J_{1}(\beta a) \\
& -4 k^{2} \alpha \beta J_{1}(\alpha a) J_{0}(\beta a)=0,
\end{aligned}
$$

where $\alpha^{2}=\left(\omega^{2} / c_{L}^{2}\right)-k^{2}, \beta^{2}=\left(\omega^{2} / c_{T}^{2}\right)-k^{2}, \omega$ is the angular velocity, $k$ is real wave number, $c_{L}$ and $c_{T}$ are the velocity of longitudinal and transverse waves in media, $a$ is the radius of the cylinder, and $J$ is Bessel function, respectively. Equation (1) is a transcendental equation with independent variables $\omega$ and $k$. Thus, a single frequency $\omega$ has more than one solution. This phenomenon corresponds well to the fact that many modes exist at a single frequency, indicating the multiple modes property of UGWs.

Phase and group velocities are two fundamental parameters that describe the characteristics of wave propagation. Phase velocity is defined as the speed of the phase of the wave propagation in the waveguide, whereas group velocity corresponds to the speed of the wave packet propagation. The curves that depict the relationships between frequency and phase velocity and group velocity are called dispersion curves. All waveguides, both symmetrical and asymmetrical, have special dispersion curves, which can be obtained via numerical calculation $[7,8]$. Other characters of guided wave propagation can be obtained in many textbooks, for example, [9-15].

For a typical steel bar in air, in which the diameter of the bar is $22 \mathrm{~mm}$, density is $7932 \mathrm{~kg} / \mathrm{m}^{3}$, compression longitudinal velocities is $5960 \mathrm{~m} / \mathrm{s}$, and transverse velocity is $3260 \mathrm{~m} / \mathrm{s}[7,16]$; the dispersive curves of phase and group velocity are shown in Figures 1 and 2, respectively.

Based on Figures 1 and 2, the following characters of UGW are drawn.

(1) More than two modes exist corresponding to a single frequency. The number of modes increases with the increasing of the frequency.

(2) Dispersion occurs in all modes, indicating that phase velocity or group velocity changes with frequency.

(3) Cut-off frequency is common in all the modes except for $L(0,1)$ and $F(1,1)$. In other words, only $L(0,1)$ and $F(1,1)$ modes can be excited when the frequency is lower than $87 \mathrm{kHz}$. This feature is very important to select the excitation frequency of the guided wave signal.

Therefore, guided waves have some important characters, such as, the capability of testing over long distances with 


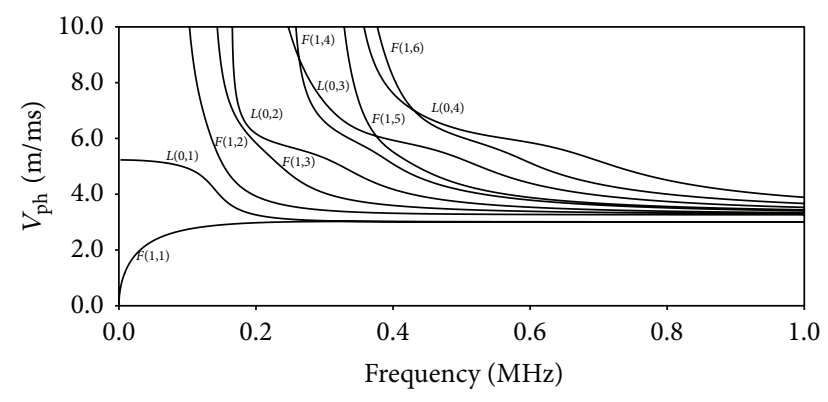

FIGURE 1: Phase velocity dispersion curves.

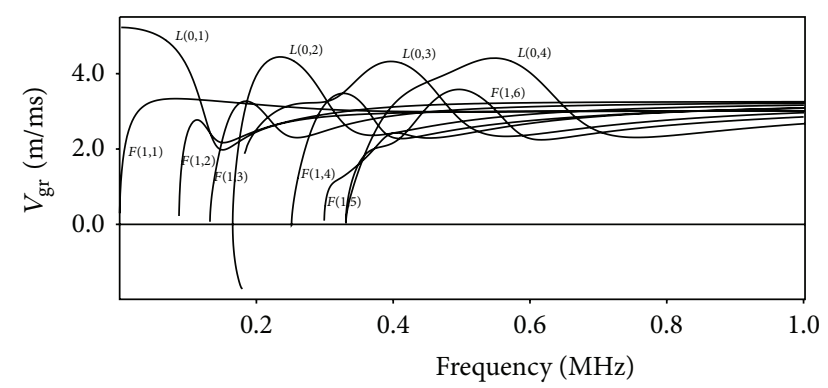

FIGURE 2: Group velocity dispersion curves.

a greater sensitivity, the ability to test multilayered structures, and being relatively inexpensive due to simplicity and sensor cost [17]. Furthermore, frequency and mode tuning can be utilized for evaluation of different types of deterioration or damage because UGW s have many different modes at a single frequency that are sensitive to different defects.

Due to the above characters, the guided wave based techniques have been found to be very efficient for damage detection and condition assessments of various aerospace, civil structures, and other nondestructive testing (NDT) areas, for example, Ajay and Carlos presented a review of guided wave for structural health monitoring; Sohn et al. [18] investigated delamination detection in composites through guided wave imaging; Song et al., $[19,20]$ developed smart piezoceramic transducers for concrete structural health monitoring; Schöpfer et al. [21] proposed a mathematical analysis of the strip-element method for the computation of dispersion curves of guided waves in anisotropic layered media including a thorough analysis of the corresponding infinite-dimensional eigenvalue problem as well as a proof of the existence of eigenvalues; De Marchi et al. [22] studied an approach based on a Warped Frequency Transform (WFT) to compensate the dispersive behavior of ultrasonic guided waves, followed by a Wigner-Ville time-frequency analysis and the Hough Transform to further improve localization accuracy. Giurgiutiu [23] studied lamb wave generation with piezoelectric wafer active sensors for structural health monitoring (SHM); Zhu et al. [24] carried out studies on two concrete slabs with different debonding between the rebars and concrete. Three proposed indices were used to identify the bond loss. They are the attenuation factor, the damage index (DI) value which is based on the comparison between the original waveform and the response signal, and the damage index based on wavelet component energy (WDI) value which is based on the wavelet component energy. The experimental results show that the technique is effective to detect the single bond loss. The DI value increases when the debond length increases but the damage location has no obvious effect on DI values. The WDI value increases with the debond length and changes with the damage location; Wang et al. [25] investigated the effects of a defect's geometric parameter on the two reflection signals in pipe using guided waves and proposed a new strategy for accurate and quantitative pipeline defect characterization; Iyer et al. [26] proposed an ultrasound acoustics-based methodology that acquires depth perception and complement 2-D crack features available from the Sewer Scanner and Evaluation Technology camera for inspection of concrete pipes; Cobb et al. [27] studied the torsional guided wave attenuation in piping from coating, temperature, and largearea corrosion and obtained the experimental results that wave attenuation is a good indicator of general corrosion level with some limitation regarding coating type; Salzburger et al. [28] studied the pipe inspection with guided waves by Electromagnetic Acoustic Transducers (EMATs) which can contribute new solutions for ultrasonic testing (UT) of pipes using guided waves; Ahmad and Kundu [29] studied the influence of water flow through pipe networks for damage detection using guided waves; Beard et al. [30] used guided waves to inspect concrete reinforcing tendons and evaluated the effect of factors such as leakage and defect geometry; Zhu et al. [31] used ultrasonic guided waves for nondestructive evaluation/structural health monitoring of trusses. Other recent developments of guided waves for SHM were also discussed in a review prepared by Huang et al. [32].

This paper concentrates on the review of the studies and progress of monitoring corrosion in reinforced concrete in the last decade based on the above characters of ultrasonic guided waves

2.2. Mechanism and Feature Extraction of Monitoring Corrosion in RC Based on UGW. Concrete is alkaline due to the presence of hydroxides of calcium, potassium, and sodium $\left(\mathrm{Ca}(\mathrm{OH})_{2}, \mathrm{KOH}\right.$, and $\left.\mathrm{NaOH}\right)$. Due to the high alkalinity of concrete, the steel reinforcing bars are passivated by an iron oxide film that protects the bar. However, in the presence of oxygen, water, and aggressive ions such as chlorides steel bars are depassivated and corrosion is initiated. For the above corrosion mechanism, reinforcement corrosion can occur in a localized manner, that is, pitting, or over a larger area, that is, general corrosion, and it is dependent on a number of different parameters. As a general rule, low to medium amounts of chloride lead to more uniform attacks, whereas medium to high mounts of chloride and concrete carbonation lead to more localized attacks.

Most of current guided wave based techniques for monitoring corrosion in steel bars are based on the following three mechanisms and feature extraction.

(1) Corrosion causes the increase in bond between the reinforcing steel bars and concrete, makes the surface of steel bars rough, and therefore affects the guided wave propagation characters. 


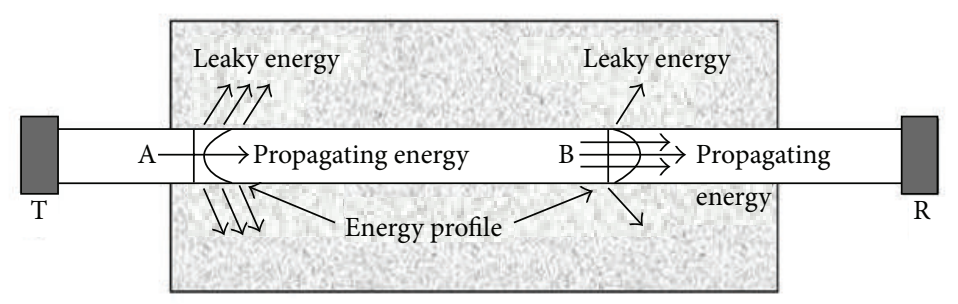

FIgURE 3: Two types of guided wave modes A and B can propagate from the transmitter T to the receiver R. From Miller et al. [33].

(2) Severe pitting is caused by corrosion and the discontinuity in steel bar affects the guided wave propagation characters.

(3) Corrosion eventually causes delamination or separation of steel rebar from the concrete affecting the strength of the propagating wave due to delamination.

Based on the above mechanism and feature extraction, most of the proposed UGW based techniques for monitoring corrosion can be categorized into four groups as: (1) monitoring corrosion induced deboning, (2) monitoring pitting corrosion, (3) monitoring corrosion induced interface roughness, and (4) monitoring combined effects in actual corrosion. The following sections review the above four groups of UGW based monitoring techniques for monitoring corrosion reinforced concrete including numerical simulation and signal processing. The influence effects such as loading condition and surface geometry of steel bar are also discussed.

2.3. Monitoring Corrosion Induced Deboning. It has been reported by Miller et al. [33] that there are two possible types of energy profiles inside the bar that can be observed in propagating guided wave modes as the wave energy travels through a steel bar from transmitter $\mathrm{T}$ to receiver $\mathrm{R}$ and these two types are denoted as modes A and B shown in Figure 3. For mode A, the energy profile is such that most of the energy propagates near the circumference of the rod; it is a surfaceseeking mode and is chosen to assess the interfacial changes. For mode B, the energy is confined near the core or central axis of the rod, it is a core-seeking mode and is suitable for detecting pitting corrosion for its low attenuation and large range of detection. Once the interface condition between steel bar and concrete changes, the energy propagation may change as well.

$\mathrm{Na}$ et al. [34] used both high (1 MHz) and low frequencies $(150 \mathrm{KHz})$ to study the effect of various bond levels by surrounding the rebar with a PVC pipe in RC beams and the effect of debonding location on the received waveforms. They in the same way conducted a comparison of steel concrete interfaces and glass fiber polymer-concrete interfaces using the guided waves $[35,36]$.

Reis et al. [37] used the fundamental flexural mode below $250 \mathrm{kHz}$ for estimation of corrosion damage in steel reinforced mortar. Debonding defects were simulated by wrapping a tape around the rebar. Waveform energy that is indicative of attenuation was used to relate to corrosion damage.
Loss of bond between steel and surrounding concrete could be detected and evaluated by this method.

Wu and Chang $[38,39]$ used the piezoelectric discs as sensors and actuators to detect debonding in reinforced concrete structures. A set of reinforced concrete square beam specimens with various bond levels were built and tested using guided mechanical waves at lower frequencies. The concrete specimen was $508 \mathrm{~mm}$ long with a $19 \mathrm{~mm}$ diameter bar. A PVC pipe was wrapped in the middle portion of the bar to simulate debonding damage.

He et al. [40] used frequencies between 1 and $2 \mathrm{MHz}$ to relate the effect of debonding on signals in cylindrical specimens. Specimens simulating 0\%, 25\%, 33\%, 50\%, 75\%, and $100 \%$ debonding were created. Bond was inhibited between the steel and concrete by surrounding the rebar with highdensity polyethylene for the necessary length prior to embedment.

$\mathrm{Li}$ et al. [41] used five-cycle sinusoidal signals with $120 \mathrm{kHz}$ central frequency to investigated the UGW energy attenuation on the different debonding levels between steel bar and concrete in both time-domain and frequencydomain analyses. Delamination was simulated by using PVC pipes.

All the above test results indicated that both in time domain and frequency domain, the received waveform is less attenuated with the increase in debonding for both low and high frequencies. However, the lower frequencies showed more sensitivity to the change in bond. There was no significant change in the waveform arrival time reported. The location of debonding is not discernible through pulse transmission as reported by Evin et al. [42, 43].

Wang et al. [44] conducted the debonding damage assessment in RC structures by numerical simulations. They developed a concrete-steel spectral element in the frequency domain to analyze wave propagation along a steel rebar in concrete. Scalar damage parameters characterizing changes in the interface (i.e., debonding damage) were incorporated into the formulation of the spectral finite element that is used for damage detection of reinforced concrete structures. A reinforced concrete beam with embedded piezoelectric elements was carried out to verify the developed spectral element method (SEM) for modeling wave propagations. Effects of the debonding damage and material properties were also studied to evaluate the effect of different damage scenarios on wave propagation in the reinforced concrete structures. The results showed that the modeling can be used to predict the wave propagation along the steel bar in 
concrete with complex interaction between the steel rebar and concrete.

Lu et al. [45] evaluated simulated corrosion in a form of partial removal of material from the rebar embedded in the concrete by attaching rectangular piezoelectric zirconatetitanate transducer (PZT) patches at the exposed ends of the rebar. In consideration of the inevitable discrepancies in different concrete beams due to specimen preparation and sensor installation, principal component analysis (PCA) based on statistical parameters extracted from wave signals was applied to highlight the difference between benchmark and damaged rebar. The results showed that PCA is capable of reducing the dimension of a complex set of original data, whose characteristics can be represented and highlighted by the first few mapped principal components. The different rebar conditions can be classified with the assistance of PCA, in terms of the existence of damage and its corresponding severity.

2.4. Monitoring Pitting Corrosion. Valor et al. [46] presented two Markov chain models to simulate pitting corrosion. The first model describes the time evolution of pit depths. The second Markov chain pitting corrosion model gives account for the maximum pit depths. Both models have been developed and validated using experimental pitting corrosion data.

In the technique of pulse echo, when there is an interface such as a crack, void, or flaw in the wave path, part of the energy is reflected back from the interface and received by the same transmitting transducer. From the display, the time of flight between the excitation and reflected pulse is measured. Knowing the group velocity of the excited longitudinal wave mode in the steel bar, the location of the defect can be calculated as follows [47]:

$$
D=\frac{V t}{2}
$$

where $D$ is the distance of defect from transducer end, $V$ is the group velocity of excited mode, and $t$ is time of flight.

Pavlakovic et al. [48] conducted the monitoring of localized corrosion in the reinforcement by creating $2 \mathrm{~mm}$ and $4 \mathrm{~mm}$ deep saw cuts (perpendicular to the bar axis) in a solid bar $8 \mathrm{~mm}$ in diameter and then casting in grout. A pulseecho arrangement was used for the saw cut reinforcement specimens, with the discontinuity located $450 \mathrm{~mm}$ away from the transmission site. A longitudinal guided wave at $3.75 \mathrm{MHz}$ was transmitted, with the results showing that wave reflections are clearly detectable from both saw cuts.

Di Scalea et al. [49] created a $2 \mathrm{~mm}$ saw cut in a sevenwire steel strand specimen located in an anchored region undergoing tensile loading. By using a through transmission test setup, it was found that the attenuation of the waveform increased for the saw cut specimen as compared to an undamaged specimen.

Sharma and Mukherjee [47] used $L(0,7)$ mode at $1 \mathrm{MHz}$ frequency to monitor notch defect in steel bars in concrete simulating pitting phenomena caused by corrosion. Both pulse echo and pulse transmission techniques were used to monitor corrosion. Appearance of notch echo indicated presence of notch in the embedded bar. By knowing the time of flight of this echo, the location of the damage can be computed by (2). It was observed that the amplitude of notch echo increased with the increase in the notch dimensions, However, the notch echo peak did not rise perceptibly even at $20 \%$ damage. Thus, its discernibility to small notches is not very high due to the nature of the mode $L(0,7)$ used for testing. As the energy of the mode is concentrated at the centre of the bar, the mode is not sensitive to surface irregularities. However, the notch location is not discernible through pulse transmission, but as the percent of damage increased from $0 \%$ to $60 \%$, the magnitude of the transmitted peak reduces. This is because as the notch dimensions increased, more energy is reflected back and less of it travels through the bar to reach the other end. Hence, relative signal attenuation of the transmitted pulse can relate to the extent of the damage in the bar.

Precise measurement of time of the flight (TOF) of the propagating signal plays a pivotal role in structure damage localization. Dai and He [50] applied a time-frequency analysis method, Wigner-Ville Distribution (WVD), to calculate the TOF of signal based on its excellent time-frequency energy distribution property. Experiments for damage localization of one-dimensional and two-dimensional structures have been studied. The results showed that the WVD-based method has better performance on the accuracy and the stability of damage localization in one-dimensional structure in comparison with traditional Hilbert envelope and Gabor wavelet transform methods.

As an incident guided wave is hitting an obstacle such as pitting in the steel bar due to corrosion, not only the same type of wave is reflected and transmitted but also mode conversion occurs at discontinuities. This mode conversion is quantified in terms of reflection and transmission, or, scattering coefficients. Gaul et al. [51] analyzed reflection and transmission of guided waves at arbitrarily shaped discontinuities in the cylinders using finite element and boundary element methods. The numerical results were confirmed with a series of laser-based ultrasound measurements. There was a good agreement between numerical and experimental results.

2.5. Monitoring Corrosion Induced Interface Roughness. Miller et al. [52] corroded rebar specimens to different levels to simulate the general corrosion, which were conducted by impressed current, removed some of the corrosion using sandpaper or wire brush, and then embedded into concrete. The diameter of the steel rebar was $22.23 \mathrm{~mm}$ and the specimen size was $12.7 \mathrm{~cm} \times 12.7 \mathrm{~cm} \times 60.96 \mathrm{~cm}$. Two corroded steel rebars removed some of the corrosion by 100 -grid sandpaper to simulate the "medium corroded sample". Another two rebars were simulated as "least corroded sample" by using the wire brush to further remove the corrosion. Tone-burst pulses were invoked at $1 \mathrm{MHz}$. The results indicate that the wave is more attenuated as the corrosion level increases. This was attributed to better bonding between the corroded steel surface and concrete, allowing more energy leakage.

Gaydeck et al. [53] embedded a $7 \mathrm{~mm}$ steel wire into a concrete mix with a $4 \%$ calcium chloride solution added to induce corrosion. The reinforced concrete specimen was 
cylindrical, with an outer diameter of $150 \mathrm{~mm}$ and a length of $1 \mathrm{~m}$. The steel wire was exposed on either end of the concrete to allow for transducer coupling. After the concrete had initially cured, a transducer with a $200 \mathrm{kHz}$ resonance was used to invoke a longitudinal wave (single-cycle pulse). Six months later, after slight corrosion occurred, another wave was sent into the specimen. The higher frequency content was most affected (i.e., loss in signal strength) by the corrosion product accumulation. This was attributed to the corrosion pressure creating better acoustic coupling at the interface between the steel and concrete, thereby allowing more energy leakage.

\subsection{Monitoring Combined Effects in Actual Corrosion.} Sharma and Mukherjee [54] studied the different modes of guided wave in the steel bar embedded in concrete. The dispersion curves show that the $L(0,1)$ mode at a low frequency of $100 \mathrm{KHz}$ is of the significant amount of axial displacement at the steel/concrete interface, so it is a surface-seeking mode and is chosen to assess the interfacial changes. It also shows that the energy of $L(0,7)$ mode at a high frequency of $1 \mathrm{MHz}$ is concentrated in the central core of the bar. Hence it is a coreseeking mode and is suitable for detecting pitting corrosion for its low attenuation and large range of detection, as shown in Figure 2.

Furthermore, the different progressions of rebar corrosion in concrete in chloride and oxide environments were conducted respectively by the surface- and core-seeking guided wave modes $[2,54]$. The above two modes were used to monitor beams undergoing accelerated impressed current corrosion. The results showed that pitting and nonuniform area loss were highlighted by severe signal attenuation in chloride corrosion with core-seeking mode. With surfaceseeking mode, the signal strength of chloride corrosion initially rises followed by the drop. This indicates that chloride corrosion starts with delamination followed by local loss of material. In oxide corrosion, the rate of corrosion is slow and localized marked by initial drop in signal due to pressure buildup by formation of corrosion products and then slow bond deterioration as depicted by gradual rise in signal strength in surface-seeking mode. Thus effective combination of surface-seeking and core-seeking of guided wave modes can relate to the differences in corrosion mechanisms and rates in the two environments in RC structures; however, the simulation of chloride corrosion as delamination is not realistic. A judicious combination of notching and delamination would be more effective for simulating chloride corrosion.

Ervin and Reis [42] and Ervin et al. [55] used both low $(<200 \mathrm{KHz}, L(0,1)$ mode) and high $[2-9 \mathrm{MHz}, L(0,9)$ mode) guided longitudinal modes for monitoring corrosion in reinforced mortar by the accelerated corrosion experiments. It was found that surface-seeking modes were sensitive to the combined effect of bond deterioration and mortar stiffness reduction while core-seeking modes related well to change in cross-sectional area of the reinforcement.

During the corrosion progression, both phenomena of increasing surface roughness and increasing separation between steel and concrete can occur simultaneously or at different stages of corrosion. Their contribution to energy propagation of guided waves is opposite to each other, so the net signal strength may either increase or decrease depending on which effect is stronger at that time, therefore a change in the signal strength does not necessarily always imply increasing corrosion. To avoid the perplex problem, Miller et al. [33] proposed a new guided wave-based technique to monitor corrosion in reinforced concrete by studying the change in the time of flight (TOF) of propagating wave in loaded reinforced concrete structures at various levels of corrosion. RC beam specimen was conducted by accelerated corrosion and subjected to bending loads at different corrosion levels. The results showed that the degree of corrosion is related to the change in the TOF of propagating waves as the RC beam is loaded laterally. The main advantage of the proposed approach is that the test result is independent of energy propagation of guided waves. In addition, it is not affected by the bonding condition between the sensors and structure.

\subsection{Influence Factors of UGW Based Monitoring for Corrosion} in $R C$. Effect of loading conditions, temperature, concrete strength, reinforcing ribs, and anchorages on the guided waves has been reported.

$\mathrm{Wu}$ and Chang $[38,39]$ investigated tension test on the rebar to determine whether loading would have any effect on the guided wave characteristics. The results showed that the applied load does not affect the wave amplitude for the frequency range and mode tested. However, the time of arrival of the waveforms did change once yielding occurred due to the rapid increase in length. They also investigated how guided wave characteristics are affected by bending. Longitudinal modes were invoked using 5-cycle tone bursts, primarily around $90 \mathrm{kHz}$. As the load was increased during a four-point bending test, a crack, perpendicular to the axis of the reinforced concrete specimen, formed in the tension zone and extended up towards the neutral axis. The results showed that as the applied loads and cracking of the surrounding concrete matrix increased, the amplitude of the waveform increased.

Li et al. [41] used Ansys software to simulate the propagation of guided waves in the rebar and explore the characteristics of ultrasonic guided wave in the steel rebar waveguide. Then two-dimensional fast Fourier transform was used to process the numerical results and to evaluate the debonding damage. The results showed that both the group velocity and the amplitude of longitudinal modes were not very sensitive to stress and temperature variations. However, the received UGW signal energy decreased with the increasing concrete strength.

For the effects of reinforcing ribs and anchorages, it was concluded that [56] (1) wave propagation is largely unaffected by the presence of surface features when the ratio of the wavelength to the surface feature dimensions is large, and there was particular sensitivity to diagonal rib patterns compared to an orthogonal axisymmetric rib pattern; (2) specimens without stirrups had stronger waveform amplitudes than specimens with stirrups.

\section{Fiber Bragg Grating (FBG) for Monitoring Corrosion in RC}

Fiber optic sensors have a number of advantages over conventional sensors, such as high sensitivity, immunity to electromagnetic interference, corrosion resistance, small-volume, 


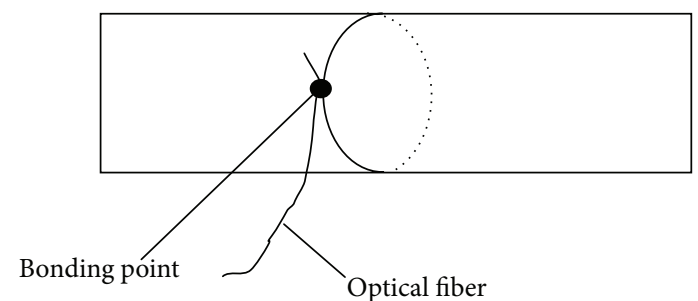

FIGURE 4: Optical fiber grating bound on surface of steel cylinder.

negligible weight, variable form, and one-line multipoint monitoring. Thus, they are valuable in structural health monitoring. Kundu et al. [57] proposed a general review of their applications in structural health monitoring. Hou et al. [58] proposed an improved negative pressure wave method based on FBG based strain sensors and wavelet analysis. Compared to conventional pressure sensors, FBG based strain sensors have favorable properties, such as high sensitivity, cheap cost, and ease of installation. Furthermore, a wavelet transform based method for identifying the pressure drop points within the FBG signals was proposed to calculate the leak position.

Many studies on FBG for monitoring corrosion are emerging in recent years. Majumder et al. [59] proposed a structural corrosion-monitoring sensor based on a pair of prestrained fiber Bragg gratings which will cause the prestrain to gradually relax when the target structure becomes corroded. Lee et al. [60] proposed a fiber optic corrosion sensor to detect rebar corrosion which was made of one fiber Bragg grating (FBG) sensor and twin steel rebar elements packaged up with concrete. Gao et al. [61] used an $80 \%$ etched-cladding fiber Bragg grating sensor to monitor the production of corrosion waste in a localized region of the rebar. Abu Hassan et al. [62] modification of the wavelength-strain coefficient of FBG for the more accurate prediction of steel bar corrosion embedded in concrete. Cheng and Dong [63] presented a new methodology to corrosion detection in reinforced concrete structures, by combining fiber Bragg grating (FBG) sensors with the electrochemical and physical properties of rebar in a simplified assembly. Ali-Alvarez et al. [64] proposed and built a simple noncontact force sensor based on an optical fiber Bragg grating attached to a small magnet for corrosion measurement. Pacheco and Bruno [65] proposed a novel long-period fiber grating (LPFG) sensor coated with a thin film of nano iron and silica particles for corrosion monitoring.

Some researches on monitoring corrosion in RC by FBG have been done by the authors of this paper. After continuous improvement a new corrosion sensor based on FBG is ultimately used in civil infrastructures. The following section reviews the studies and application by the authors $[66,67]$.

3.1. Mechanism of the FBG Based Corrosion Sensor. FBG is obtained by producing a periodic variation in the index of refraction along the short sections (about $10-20 \mathrm{~mm}$ ) in the core of an optical fiber. If it is fixed on the surface of a steel bar, the variation in the volume due to corrosion can be measured by monitoring the wavelength shift of the FBG.

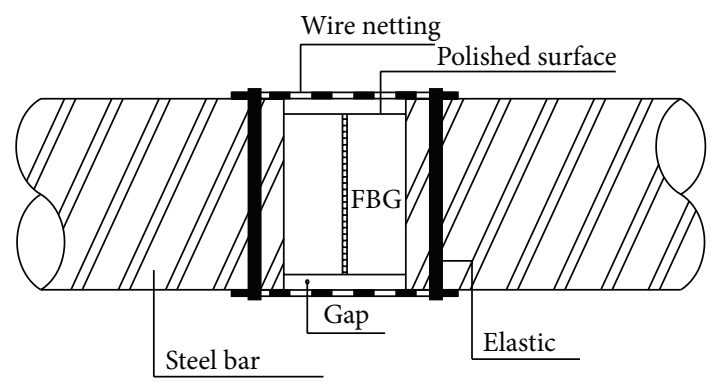

FIGURE 5: The scheme of package.

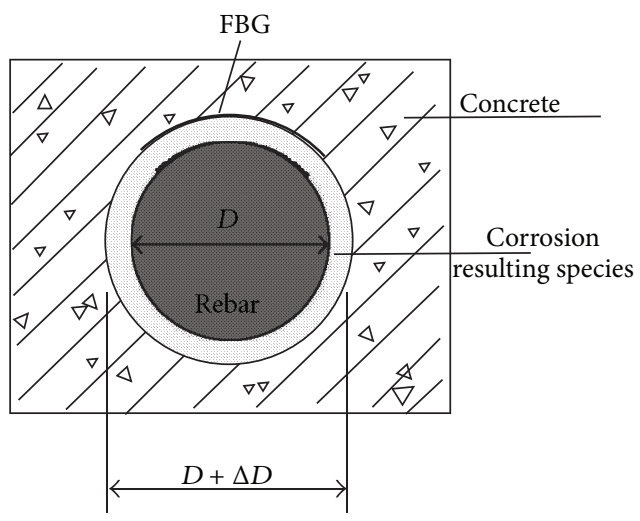

FIGURE 6: FBG strain.

The corrosion intensity can be described by the wavelength shift of FBG as follows [67]:

$$
\begin{aligned}
\rho & =\frac{\Delta V}{V}=\frac{(D+\Delta D)^{2}-D^{2}}{D^{2}} \\
& =\left(1+\frac{\Delta D}{D}\right)^{2}-1=(1+\varepsilon)^{2}-1 \\
& =\left(1+\frac{\Delta \lambda_{b}}{\kappa_{\varepsilon}}\right)^{2}-1,
\end{aligned}
$$

where $D$ is the original diameter of steel rebar, $\Delta D$ is the diameter increment, $\Delta \lambda_{b}$ is the wavelength shift of FBG, $\kappa_{z}$ is the strain sensitivity of FBG, and $\rho$ is the increase rate of the steel volume.

3.2. Design of FBG Corrosion Sensor. The corrosion sensor using FBG is designed in the following procedures: (1) Mechanically polish the surface of a $20 \mathrm{~mm}$ part of reinforcement made of hot rolled steel; (2) enwind FBG one coil on the polished surface of the reinforcement tightly and then bind it through the epoxy binder at the junction as shown in Figure 4 ; (3) put one piece of wire netting above the polished area to prevent FBG from being destroyed during concrete pouring which is fixed by the elastic on two ends as shown in Figure 5.

The FBG can expand freely without constraint from the protective layer because there is $2 \mathrm{~mm}$ gap between them. Water and air can reach the steel rebar at the packaged region through the holes on the wire netting, so it is in the same corrosion condition as others. As shown in Figure 6, when 


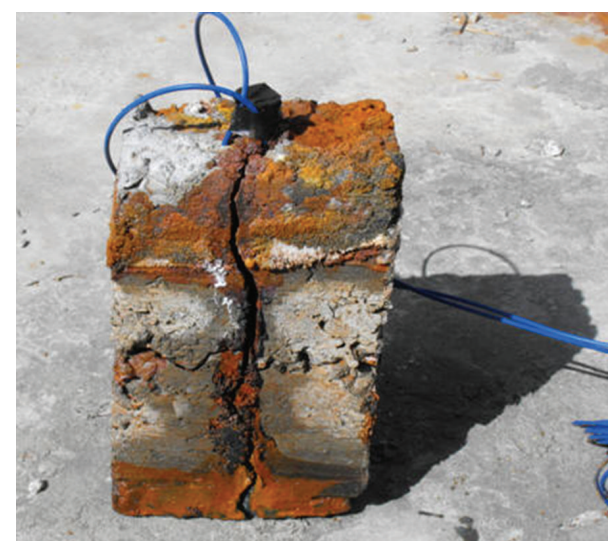

FIGURE 7: Cracking of concrete block.

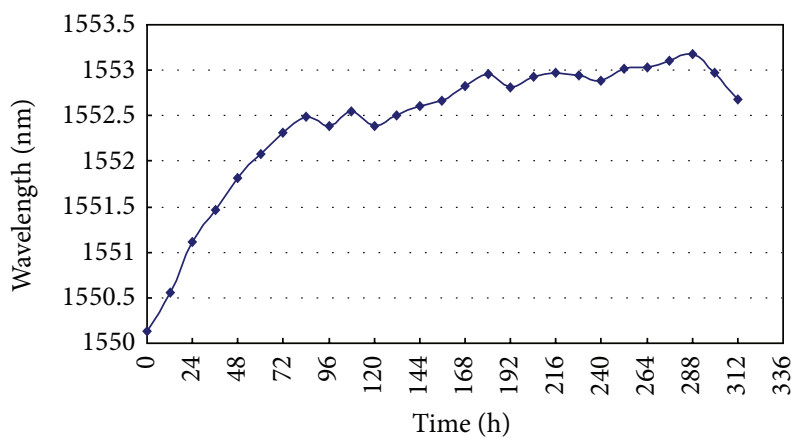

FIGURE 8: Wavelength of FBG versus elapsed time.

the reinforcement is corroded within the concrete structure, the resulting species deposits continuously on the surface of the rebar because the loss of mass is hindered by concrete, the volume of the steel rebar increases during corrosion, and the coverage concrete is finally cracked. During the corrosion, the tensile strain in the FBG is increased with the increasing diameter of steel reinforcement. Finally, the tensile stress in the FBG can reach the level to break FBG; the corrosion sensor then becomes invalid.

Experimental tests on the designed FBG corrosion sensor were carried out by accelerated corrosion condition and some vessels were embedded from outer side of the specimen to the polished area to make the position where sensor was attached easier to be corroded. As shown in Figure 7, substantial cracks occurred in the specimen. The results obtained from the sensor were analyzed. It can be observed from Figure 8 that the wavelength shift increases gradually with time, and even the curve is fluctuated in some parts, the overall trend is increased. Evidently, the curve can be divided into three parts. In the first part, that is, from point A to B, the wavelength shift is increased rapidly with time due to the voluminous rust product accumulation, which is a consequence of the concrete pores adjacent to the rebar. In the second part, that is, from point $B$ to $C$, there is only relatively small increase in the curve with some fluctuations, which is due to the fact that concrete is further compacted and micro cracks are occurring. In the last part, the wavelength shift is declined at turning point $\mathrm{C}$ because of the appearance of large cracks, and

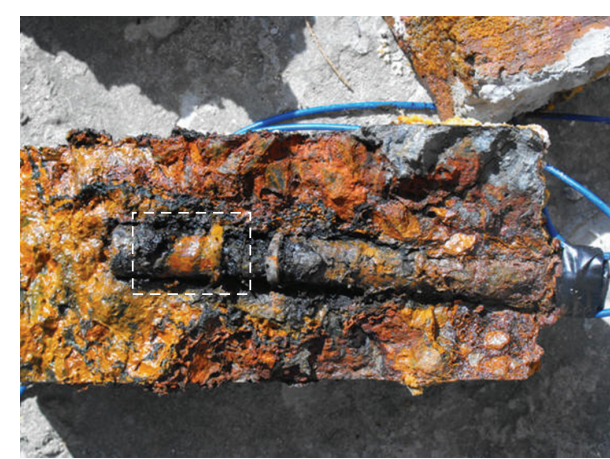

Figure 9: Corroded bar from FBG sample.

the resulting species dissolved into the solution, and the volume of the steel reinforcement decreased during corrosion.

To confirm the results, a commercial device named CANIN (type: EN-0503) which was based on half-cell corrosion potential method was used to monitor the corrosion. It was noted from the test that when the wavelength changed from $1550 \mathrm{~nm}$ to $1554 \mathrm{~nm}$, the potential read from the CANIN was between 500 and $400 \mathrm{mv}$, which verifies the appearance of light corrosion. It can be observed from Figure 9, that there is a significant difference between the polished region and the other region of the bar, which indicates the occurrence of corrosion in the area where FBG is attached (marked by the dashed line in the figure).

The lab experimental results indicated that the FBG has a clear difference in reading precorrosion and postcorrosion data, and the monitoring data are reliable to evaluate the corrosion. Thus, the designed FBG based corrosion sensors can be used for monitoring corrosion of steel reinforcements in concrete structures.

3.3. Application of the Designed FBG Corrosion Sensors in Xiamen Xiang'an Subsea Tunnel in China. Xiamen Xiang'an Subsea Tunnel in China is located in eastern part of Xiamen island and is connecting Xiamen island and the Xiang'an mainland as shown in Figure 10. It is the largest cross-section subsea tunnel in China with a total length of $8.695 \mathrm{~km}$. The deepest part of the tunnel is 70 meter below the sea level. The construction of the tunnel was started in September 2005 and finished in April 2010.

The FBG based corrosion sensors developed by the authors were made of the same steel as the practical engineering and applied in the Xiamen Xiang'an Subsea Tunnel. Corrosion sensors were installed at the apex and haunches of dangerous section as shown in Figure 11.

Additionally, to compensate effects of temperature variations on the shift of wavelength, another FBG sensor encapsulated in a polyvinyl chloride (PVC) pipe was arranged in parallel nearby freely to monitor the wavelength shift resulted from temperature variation, as shown in Figures 12, 13, 14 and 15.

Other embedded corrosion sensors were installed to compare the accuracy of data, including the commercial multiprobe CorroWatch sensor and ERE20 reference electrode produced by Force company in Denmark. 


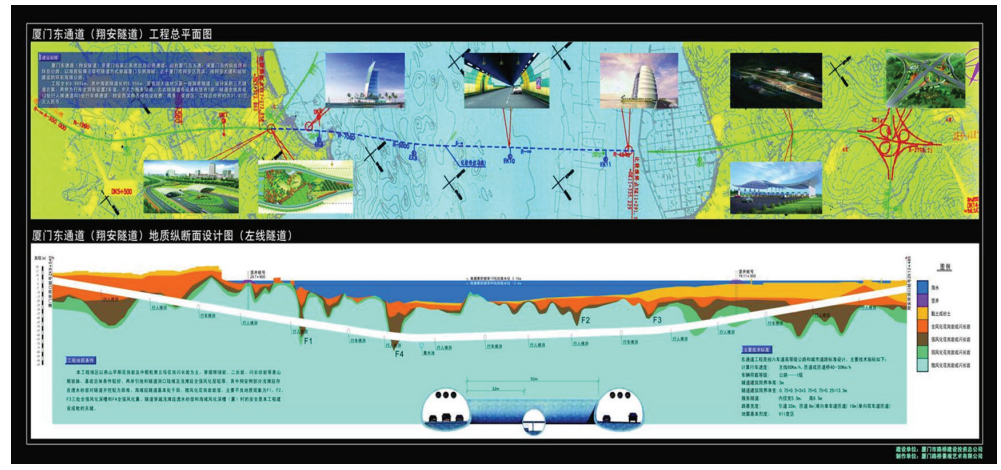

Figure 10: General plan and profile view of Xiang'an Subsea-tunnel.

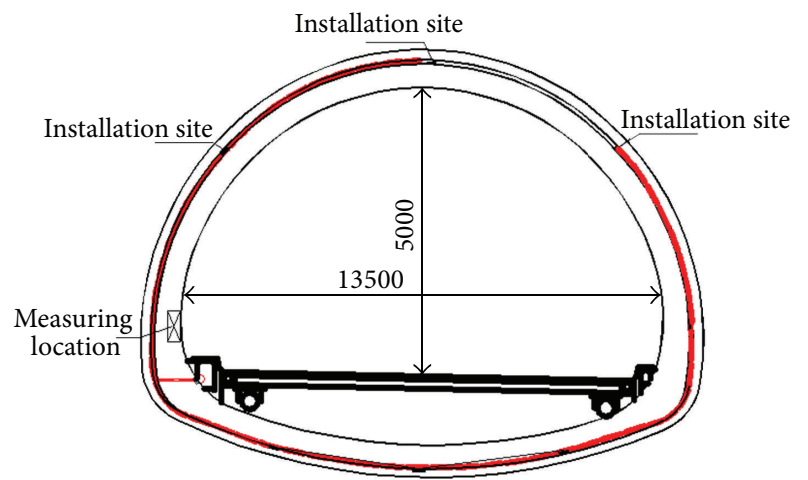

FIGURE 11: Section view and installation site in Xiangan Sub-sea Tunnel.

Twenty FBG sensors were installed in different positions of the tunnel. After concreting, sixteen sensors survived. Monitoring data have been collected since April 2010. So far, the results obtained from the sensors are shown in Figure 16. It can be observed that all surviving sensors are still in work and there is no obvious corrosion in monitoring areas of the tunnel. The data from other electrochemical sensors at the same position also demonstrated that corrosion has not started yet. Data from all the sensors will be collected for the next several years and will be analyzed to develop a detailed knowledge-based corrosive behavior of the rebars.

\section{Introduction of Some Other Physical Techniques for Monitoring Corrosion in RC}

There are some other physical techniques for monitoring steel corrosion. These techniques are briefly reviewed as follows.

4.1. Electrical Resistance Probe. Electrical resistance (ER) probe is one specific type of embedded sensor which is simple to be used for monitoring corrosion. In the technique, resistance probes made of the same material with steel bar are first embedded in concrete, and then the resistance of probes can be measured by balancing bridge which is inversely proportional to its cross-sectional area. Therefore, the thickness reduction due to corrosion can be estimated from the change in resistance of probes so as to achieve the test purpose $[68,69]$. Although applications of these probes in concrete are relatively rare, the results have proved that they can accurately determine the cumulative corrosion damage of steel in cementitious materials [70]. Consequently, reliable assessment of the average general corrosion rate over defined time intervals is possible [70]. On the other hand, the response of these probes to localized corrosion types and transient events is limited. ER probes are based on physical response to corrosion and are therefore not directly susceptible to variations in the electrochemical parameters. For this reason they are particularly suited for the long-term verification of the suitability of electrochemical sensors for corrosion measurements in concrete [71].

4.2. X-Ray Diffraction. X-Ray technique is based on the principle that intensity of X-Ray beams reduces while passing through a corroded material. Analysis of material composition and relative amounts of corrosion products can be achieved by the technique. It is simple but hazardous [72-75].

4.3. Vortex and Magnetic Flux Leakage. Steel bar reaches magnetic saturation with the electromagnetic devices placed on the surface of concrete. Abnormal phenomenon in magnetic field will appear due to loss in cross-sectional area of steel bar caused by corrosion. Loss rate of the cross-sectional area of reinforcement can be determined with analysis of these exceptions. It is an effective method to quantitatively detect the loss of reinforcement with accuracy and it is nondestructive [76-80].

4.4. Acoustic Emission. Yuma et al. studied corrosion mechanisms in reinforced concrete by acoustic emission (AE) [81]. During corrosion in reinforcement concrete, the surrounding concrete will crack due to the expansion stress resulted from the corrosion products. When it is cracking, part of energy will release in the form of sound waves. Therefore position and strength of the emission source can be detected by acoustic emission sensors. It is hard to avoid interference with other waves, so it is difficult to establish the relationship between the level of reinforcement corrosion activity and the intensity of acoustic emission [82]. Calabres et al. [83, 84] recently 


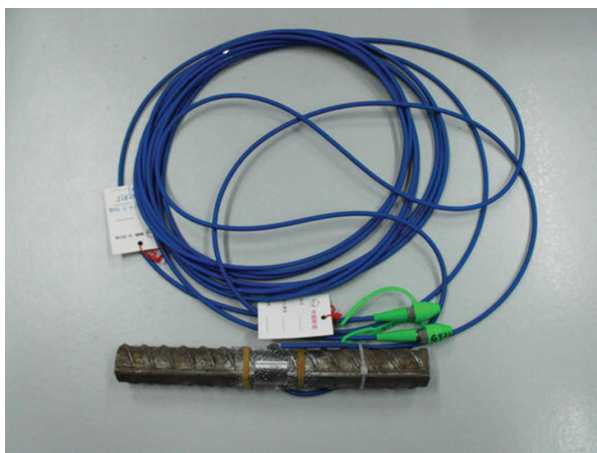

(a)

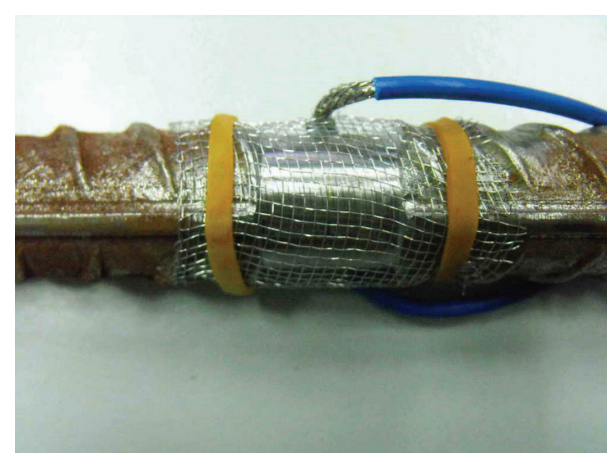

(b)

FIGURE 12: Fiber optic corrosion sensor.

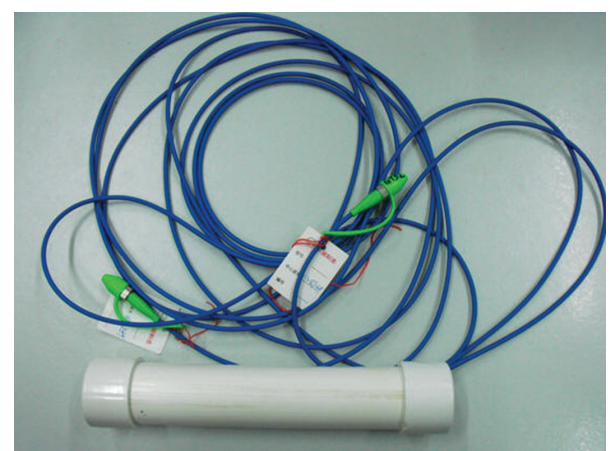

FIGURE 13: Reference FBG sensor for temperature compensation.

studied noise removal and univariate and multivariate statistical analysis in AE corrosion monitoring of steel reinforcement in concrete. Kundu et al. [57] used a combination of a PZT and an electromagnetic acoustic transducer (EMAT) to inspect the interface between steel bar and concrete, such that arrangement was made to overcome the major shortcoming of PZT and EMAT. Elfergani et al. [85] reported on using the acoustic emission (AE) technique to detect and locate the early stages of corrosion and macrocracks and furthermore classify different crack types to aid maintenance priorities. Benedetti et al. [86] presented an accelerated corrosion and continuous AE monitoring test. The effectiveness of $\mathrm{AE}$ in detecting and characterizing the initiation of the corrosion process is discussed on the basis of results from small-scale, precracked RC specimens that are representative of areas near the clear cover in typical RC members. They proposed a new approach of $\mathrm{AE}$ data interpretation based on time-driven parameters.

\section{Conclusions}

In this paper, some physical based monitoring techniques for condition assessment of steel corrosion in reinforced concrete in the last decade are reviewed. Especially, techniques based on ultrasonic guided wave (UGW) and Fiber Bragg grating (FBG) are emphasized as these two important techniques have many advantages for monitoring corrosion in reinforced concrete. Also, FBG corrosion sensors developed by the authors with its application are presented.

From the review, it can be seen that physical based monitoring techniques can conduct accurate condition assessment of steel corrosion through direct measurements on embedded steels. Compared with the conventional monitoring techniques based on electrochemistry, physical based monitoring techniques not only provide alternative tools for monitoring steel corrosion in concrete bust also have the distinguished superiority as electrochemistry techniques can only assess corrosion through monitoring the surrounding concrete medium.

It is addressed in this review that UGW based monitoring techniques are promising for the accurate condition assessment of steel corrosion in reinforced concrete over a larger inspection range compared with other techniques. However, there are still difficulties in the practical application of UGW based techniques for the condition assessment of corrosion in reinforced concrete. The main challenges and future development trends of UGW based techniques are as follows.

(1) Most of the current researches are concentrated on monitoring individual phenomenon of interface roughness, deboning, and steel pitting induced by corrosion in reinforced concrete. Although much progress has been made, accurate detection the range of interface roughness and deboning and the location of steel pitting is still challenging. Moreover, it is imperative to develop the efficient techniques for monitoring the combined effects induced by the actual steel corrosion concrete.

(2) One more difficulty of UGW based technique for monitoring corrosion in reinforced concrete is the limitation of monitoring range for certain modes and frequencies. Unlike guided wave propagation in other multilayered systems, such as a metal pipeline in air, wave energy in steel bars embedded in mortar or concrete is lost (i.e., attenuated) at high rates due to leakage into the surrounding concrete. Generally, modes exist that have attenuation minima, or dips, with minimal amounts of wave energy lost due to leakage 


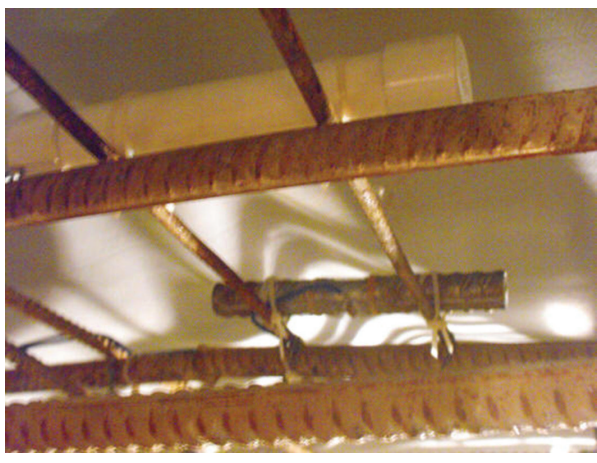

(a)

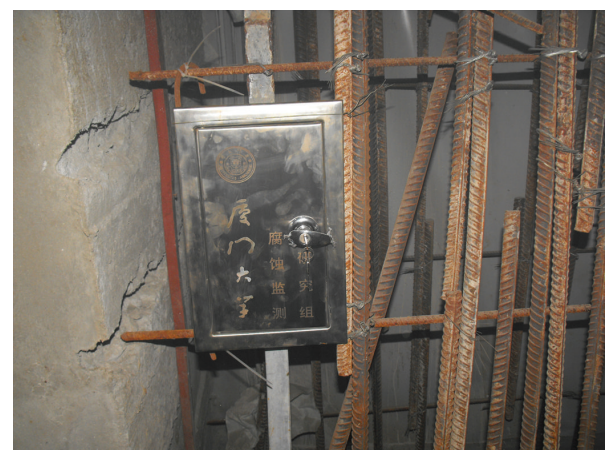

(b)

FIgURE 14: Photographs of field application.

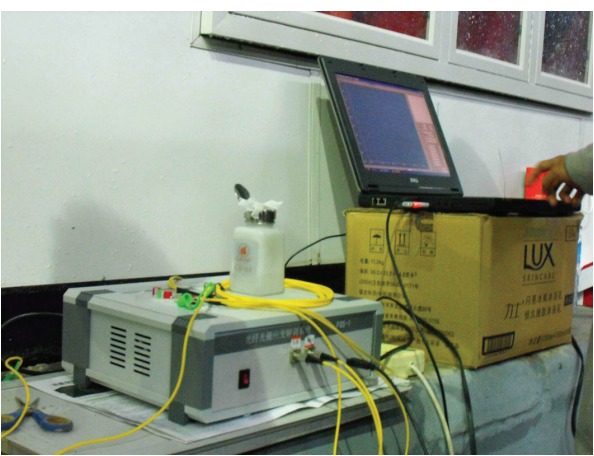

(a)

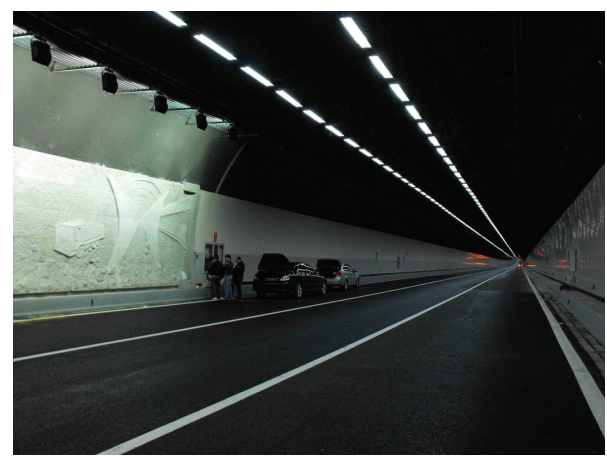

(b)

FIGURE 15: Photographs of testing in situ.

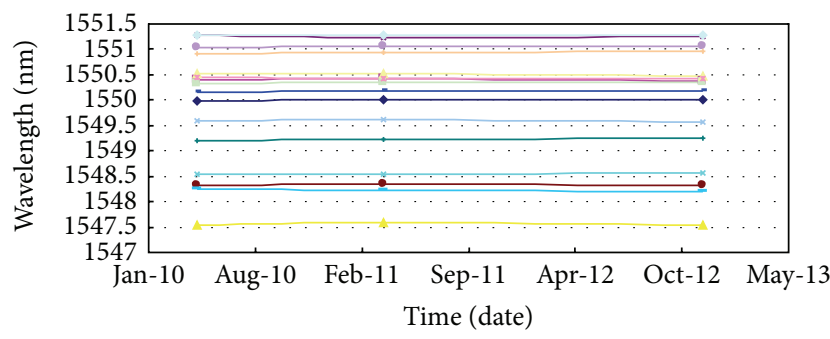

FIGURE 16: Wavelength of FBG versus elapsed time in the tunnel.

and material absorption. So the successful development of UGW monitoring techniques require careful model studies to be performed based on properly understanding the nature of the waves and knowledge of the attenuation.

(3) The increase of steel corrosion level in an RC structure can either increase the recorded wave strength because of increasing separation between steel and concrete or decrease the strength of the recorded guided waves due to increasing surface roughness. During the corrosion progression, both phenomena can occur simultaneously or at different stages of corrosion, the net signal strength may either increase or decrease depending on which effect is stronger at that time or may remain unchanged if the two opposing effects cancel each other. This is another difficulty associated with the current guided wave-based techniques. To study which effect dominates, propagation mechanism, factors, and regular pattern of attenuation of guided waves along steel bar embedded in concrete are very important. These are future research trends.

It is also noted that monitoring techniques based on Fiber Bragg grating (FBG) are also useful tools for condition assessment of steel corrosion in reinforced concrete. Some relevant techniques have been applied in the monitoring of $\mathrm{RC}$ structures. However, current techniques can only conduct localized inspection of corrosion. It is expected to develop distributed and long-gauge fiber Bragg grating technique for solution of this problem.

While other physical based monitoring techniques have been proposed, corrosion process can occur in multiple stages, and the mechanism of corrosion is sophistic; there is no single evaluation method to accurately detect and monitor in situ the entire corrosion process to date. It is the future development trend to combine with a variety of methods simultaneously for the accurate condition assessment of steel corrosion reinforced concrete infrastructures.

\section{Acknowledgments}

This research is supported by the National Natural Science Foundation of China (NSFC) through the Grant no. 51178406, 
the research funding SLDRCE10-MB-01 from the State Key Laboratory for Disaster Reduction in Civil Engineering at Tongji University, China, and the Key Project of Fujian province of China (no. 2013Y0079). The authors also greatly appreciate the collaboration work from Professor X. P. Dong at the school of information science and engineering of Xiamen University for the field testing of proposed FBG corrosion.

\section{References}

[1] J. P. Broomfield, Corrosion of Steel in Concrete: Understanding, Investigation and Repair, Taylor \& Francis, New York, NY, USA, 2nd edition, 2007.

[2] S. Sharma and A. Mukherjee, "Nondestructive evaluation of corrosion in varying environments using guided waves," Research in Nondestructive Evaluation, vol. 24, no. 2, pp. 63-88, 2013.

[3] H. S. Shang, T. H. Yi, and Y. P. Song, "Behavior of plain concrete of a high water-cement ratio after freeze-thaw cycles," Materials, vol. 5, no. 9, pp. 1698-1707, 2012.

[4] H.-S. Shang, T.-H. Yi, and L.-S. Yang, "Experimental study on the compressive strength of big mobility concrete with nondestructive testing method," Advances in Materials Science and Engineering, vol. 2012, Article ID 345214, 6 pages, 2012.

[5] P. Matt, "Non-destructive evaluationand monitoring of posttensioning tendons," fib Bull. 15: Durability of post-tensioning tendon, pp. 100-108, 2001.

[6] R. W. Griffiths and G. W. Nelson, "Recent and current developments in distributed fiber optic sensing for structural monitoring," in Fiber Optic and Laser Sensors VI, vol. 985 of Proceedings of SPIE, pp. 69-76, San Diego, Calif, USA, 1989.

[7] J. L. Rose, Ultrasonic Waves in Solid Media, Cambridge University Press, Cambridge, UK, 1999.

[8] J. L. Rose, "Dispersion curves in guided wave testing," Materials Evaluation, vol. 61, no. 1, pp. 20-22, 2003.

[9] J. D. Achenbach, Wave Propagation in Elastic Solids, vol. 16 of North-Holland Series in Applied Mathematics and Mechanics, North-Holland, New York, NY, USA, 1984.

[10] J. Miklowitz, The Theory of Elastic Waves and Waveguides, vol. 22 of North-Holland Series in Applied Mathematics and Mechanics, North-Holland, New York, NY, USA, 1978.

[11] C. S. Kino, Acoustic Waves: Devices, Imaging and Digital Signal Processing, Prentice Hall, Upper Saddle River, NJ, USA, 1987.

[12] B. A. Auld, Acoustic Fields and Waves in Solids, vol. 2, Kreiger Publishing, Malabar, Fla, USA, 2nd edition, 1990.

[13] K. F. Graff, Wave Motion in Elastic Solids, Dover Publications, New York, NY, USA, 1991.

[14] A. H. Nayfeh, Wave Propagation in Layered Anisotropic Media with Applications to Composites, vol. 39 of North-Holland Series in Applied Mathematics and Mechanics, North-Holland, Amsterdam, The Netherlands, 1995.

[15] J. L. Rose, "A baseline and vision of ultrasonic guided wave inspection potential," Journal of Pressure Vessel Technology, vol. 124, no. 3, pp. 273-282, 2002.

[16] Z. P. Zheng, Y. Lei, X. P. Cui, and Y. Song, "Non-destructive test of the steel bar by using piezoceramics sheets," in Proceedings of the 3rd International Conference on Advanced Measurement and Test, Xiamen, China, March 2013.
[17] T. R. Hay and J. L. Rose, "Interfacing guided-wave ultrasound with wireless technology," in Smart Structures and MaterialsSensors and Smart Structures Technologies for Civil, Mechanical, and Aerospace Systems, vol. 5391 of Proceedings of SPIE, pp. 314320, San Diego, Calif, USA, March 2004.

[18] H. Sohn, D. Dutta, J. Y. Yang et al., "Delamination detection in composites through guided wave field image processing," Composites Science and Technology, vol. 71, no. 9, pp. 1250-1256, 2011.

[19] G. Song, Y. L. Mo, K. Otero, and H. Gu, "Health monitoring and rehabilitation of a concrete structure using intelligent materials," Smart Materials and Structures, vol. 15, no. 2, pp. 309-314, 2006.

[20] R. L. Wang, H. Gu, Y. L. Mo, and G. Song, "Proof-of-concept experimental study of damage detection of concrete piles using embedded piezoceramic transducers," Smart Materials and Structures, vol. 22, no. 4, Article ID 042001, 2013.

[21] F. Schöpfer, F. Binder, A. Wöstehoff, and T. Schuster, "A mathematical analysis of the strip-element method for the computation of dispersion curves of guided waves in anisotropic layered media," Mathematical Problems in Engineering, vol. 2010, Article ID 924504, 17 pages, 2010.

[22] L. De Marchi, E. Baravelli, G. Cera, N. Speciale, and A. Marzani, "Warped Wigner-Hough transform for defect reflection enhancement in ultrasonic guided wave monitoring," Mathematical Problems in Engineering, vol. 2012, Article ID 358128, 15 pages, 2012.

[23] V. Giurgiutiu, "Lamb wave generation with piezoelectric wafer active sensors for structural health monitoring," in Smart Structures and Materials: Smart Structures and Integrated Systems, vol. 5056 of Proceedings of SPIE, pp. 111-122, San Diego, Calif, USA, March 2003.

[24] X. Q. Zhu, H. Hao, and K. Q. Fan, "Detection of delamination between steel bars and concrete using embedded piezoelectric actuators/sensors," Journal of Civil Structural Health Monitoring, vol. 3, no. 2, pp. 105-115, 2013.

[25] X. Wang, P. W. Tse, C. K. Mechefske, and M. Hua, "Experimental investigation of reflection in guided wave-based inspection for the characterization of pipeline defects," NDT \& E International, vol. 43, no. 4, pp. 365-374, 2010.

[26] S. Iyer, S. K. Sinha, M. K. Pedrick, and B. R. Tittmann, "Evaluation of ultrasonic inspection and imaging systems for concrete pipes," Automation in Construction, vol. 22, pp. 149-164, 2012.

[27] A. C. Cobb, H. Kwun, L. Caseres, and G. Janega, “Torsional guided wave attenuation in piping from coating, temperature, and large-area corrosion," NDT \& E International, vol. 47, pp. 163-170, 2012.

[28] H.-J. Salzburger, F. Niese, and G. Dobmann, "Emat pipe inspection with guided waves," Welding in the World, vol. 56, no. 5-6, pp. 35-43, 2012.

[29] R. Ahmad and T. Kundu, "Influence of water flow through pipe networks for damage detection using guided waves," in Nondestructive Testing of Materials and Structures, vol. 6 of RILEM Bookseries, pp. 681-687, Springer, Amsterdam, The Netherlands, 2013.

[30] M. D. Beard, M. J. S. Lowe, and P. Cawley, "Inspection of steel tendons in concrete using guided waves," in AIP Conference Proceedings, vol. 22 of Review of Quantitative Nondestructive Evaluation, pp. 1139-1147, Bellingham, Wash, USA, July 2003.

[31] X. Zhu, P. Rizzo, A. Marzani, and J. Bruck, "Ultrasonic guided waves for nondestructive evaluation/structural health monitoring of trusses," Measurement Science and Technology, vol. 21, no. 4, Article ID 045701, 2010. 
[32] G. Huang, F. Song, and X. Wang, "Quantitative modeling of coupled piezo-elastodynamic behavior of piezoelectric actuators bonded to an elastic medium for structural health monitoring: a review," Sensors, vol. 10, no. 4, pp. 3681-3702, 2010.

[33] T. H. Miller, T. Kundu, J. Huang, and J. Y. Grill, "A new guided wave-based technique for corrosion monitoring in reinforced concrete," Structural Health Monitoring, vol. 12, no. 1, pp. 35-47, 2013.

[34] W.-B. Na, T. Kundu, and M. R. Ehsani, "Ultrasonic guided waves for steel bar concrete interface testing," Materials Evaluation, vol. 60, no. 3, pp. 437-444, 2002.

[35] W.-B. Na, T. Kundu, and M. R. Ehsani, "Lamb waves for detecting delamination between steel bars and concrete," ComputerAided Civil and Infrastructure Engineering, vol. 18, no. 1, pp. 5863, 2003.

[36] W.-B. Na, T. Kundu, and M. R. Ehsani, "A comparison of steel/ concrete and glass fiber reinforced polymers/concrete interface testing by guided waves," Materials Evaluation, vol. 61, no. 2, pp. 155-161, 2003.

[37] H. Reis, B. L. Ervin, D. A. Kuchma, and J. T. Bernhard, "Estimation of corrosion damage in steel reinforced mortar using guided waves," Journal of Pressure Vessel Technology, vol. 127, no. 3, pp. 255-261, 2005.

[38] F. Wu and F.-K. Chang, "Debond detection using embedded piezoelectric elements in reinforced concrete structures-part I: experiment," Structural Health Monitoring, vol. 5, no. 1, pp. 515, 2006.

[39] F. Wu and F.-K. Chang, "Debond detection using embedded piezoelectric elements for reinforced concrete structures-part II: analysis and algorithm," Structural Health Monitoring, vol. 5, no. 1, pp. 17-28, 2006.

[40] C. He, J. K. van Velsor, C. M. Lee, and J. L. Rose, "Health monitoring of rock bolts using ultrasonic guided waves," in AIP Conference Proceedings, Quantitative Nondestructive Evaluation, pp. 195-201, Reston, Va, USA, 2006.

[41] D. Li, T. Ruan, and J. Yuan, "Inspection of reinforced concrete interface delamination using ultrasonic guided wave nondestructive test technique," Science China Technological Sciences, vol. 55, no. 10, pp. 2893-2901, 2012.

[42] B. L. Ervin and H. Reis, "Longitudinal guided waves for monitoring corrosion in reinforced mortar," Measurement Science and Technology, vol. 19, no. 5, Article ID 055702, 2008.

[43] B. L. Ervin, D. A. Kuchma, J. T. Bernhard, and H. Reis, "Monitoring corrosion of rebar embedded in mortar using highfrequency guided ultrasonic waves," Journal of Engineering Mechanics, vol. 135, no. 1, pp. 9-19, 2009.

[44] Y. Wang, X. Zhu, H. Hao, and J. Ou, "Guided wave propagation and spectral element method for debonding damage assessment in RC structures," Journal of Sound and Vibration, vol. 324, no. 3-5, pp. 751-772, 2009.

[45] Y. Lu, J. C. Li, L. Ye, and D. Wang, "Guided waves for damage detection in rebar-reinforced concrete beams," Construction and Building Materials, vol. 47, pp. 370-378, 2013.

[46] A. Valor, F. Caleyo, L. Alfonso, J. C. Velázquez, and J. M. Hallen, "Markov chain models for the stochastic modeling of pitting corrosion," Mathematical Problems in Engineering, vol. 2013, Article ID 108386, 13 pages, 2013.

[47] S. Sharma and A. Mukherjee, "Longitudinal guided waves for monitoring chloride corrosion in reinforcing bars in concrete," Structural Health Monitoring, vol. 9, no. 6, pp. 555-567, 2010.
[48] B. N. Pavlakovic, M. J. S. Lowe, and P. Cawley, "High-frequency low-loss ultrasonic modes in imbedded bars," Journal of Applied Mechanics, vol. 68, no. 1, pp. 67-75, 2001.

[49] F. L. di Scalea, P. Rizzo, and F. Seible, "Stress measurement and defect detection in steel strands by guided stress waves," Journal of Materials in Civil Engineering, vol. 15, no. 3, pp. 219-227, 2003.

[50] D. Dai and Q. He, "Structure damage localization with ultrasonic guided waves based on a time-frequency method," Signal Processing, vol. 96, pp. 21-28, 2014.

[51] L. Gaul, H. Sprenger, C. Schaal, and S. Bischoff, "Structural health monitoring of cylindrical structures using guided ultrasonic waves," Acta Mechanica, vol. 223, no. 8, pp. 1669-1680, 2012.

[52] T. Miller, C. J. Hauser, and T. Kundu, "Nondestructive inspection of corrosion and delamination at the concrete-steel reinforcement interface," in Proceedings of the ASME International Mechanical Engineering Congress and Exposition, pp. 121-128, New Orleans, La, USA, November 2002.

[53] P. A. Gaydeck, F. M. Burdekin, W. Damaj, D. G. John, and P. A. Payne, "Digital deconvolution analysis of ultrasonic signals influenced by the presence of longitudinally aligned steel cables in pre-stressed concrete," Measurement Science and Technology, vol. 3, no. 9, article 909, pp. 909-917, 1992.

[54] S. Sharma and A. Mukherjee, "Monitoring corrosion in oxide and chloride environments using ultrasonic guided waves," Journal of Materials in Civil Engineering, vol. 23, no. 2, pp. 207211, 2011.

[55] B. L. Ervin, J. T. Bernhard, D. A. Kuchma, and H. Reis, "Estimation of corrosion damage to steel reinforced mortar using frequency sweeps of guided mechanical waves," in Smart Structures and Materials-Sensors and Smart Structures Technologies for Civil, Mechanical, and Aerospace Systems, vol. 6174 of Proceedings of SPIE, pp. 1-12, San Diego, Calif, USA, 2006.

[56] B. L. Ervin, J. T. Bernhard, D. A. Kuchma, and H. Reis, "Estimation of general corrosion damage to steel reinforced mortar using frequency sweeps of guided mechanical waves," Insight, vol. 48, no. 11, pp. 682-692, 2006.

[57] T. Kundu, S. Banerjee, and K. V. Jata, "An experimental investigation of guided wave propagation in corrugated plates showing stop bands and pass bands," Journal of the Acoustical Society of America, vol. 120, no. 3, pp. 1217-1226, 2006.

[58] Q. M. Hou, L. Ren, W. Jiao, P. H. Zou, and G. B. Song, "An improved negative pressure wave method for natural gas pipeline leak location using FBG based strain sensor and wavelet transform," Mathematical Problems in Engineering, vol. 2013, Article ID 278794, 8 pages, 2013.

[59] M. Majumder, T. K. Gangopadhyay, A. K. Chakraborty, K. Dasgupta, and D. K. Bhattacharya, "Fibre Bragg gratings in structural health monitoring - present status and applications," Sensors and Actuators A, vol. 147, no. 1, pp. 150-164, 2008.

[60] J.-R. Lee, C.-Y. Yun, and D.-J. Yoon, "A structural corrosionmonitoring sensor based on a pair of prestrained fiber Bragg gratings," Measurement Science and Technology, vol. 21, no. 1, Article ID 017002, 2010.

[61] J. Gao, J. Wu, J. Li, and X. Zhao, "Monitoring of corrosion in reinforced concrete structure using Bragg grating sensing," NDT \& E International, vol. 44, no. 2, pp. 202-205, 2011.

[62] M. R. Abu Hassan, M. H. Abu Bakar, K. Dambul, and F. R. Adikan, "Optical-based sensors for monitoring corrosion of reinforcement rebar via an etched cladding Bragg grating," Sensors, vol. 12, no. 11, pp. 15820-15826, 2012. 
[63] W. Chen and X. Dong, "Modification of the wavelength-strain coefficient of FBG for the prediction of steel bar corrosion embedded in concrete," Optical Fiber Technology, vol. 18, no. 1, pp. 47-50, 2012.

[64] S. Ali-Alvarez, P. Ferdinand, S. Magne, and R. P. Nogueira, "Corrosion detection and evolution monitoring in reinforced concrete structures by the use of fiber Bragg grating sensor," in Sensors and Smart Structures Technologies for Civil, Mechanical, and Aerospace Systems, vol. 8692 of Proceedings of SPIE, San Diego, Calif, USA, April 2013.

[65] C. J. Pacheco and A. C. Bruno, "A noncontact force sensor based on a fiber Bragg grating and its application for corrosion measurement," Sensors, vol. 13, no. 9, pp. 11476-11489, 2013.

[66] Y. Huang, Z. Gao, G. Chen, and H. Xiao, "Long period fiber grating sensors coated with nano iron/silica particles for corrosion monitoring," Smart Materials and Structures, vol. 22, no. 7, Article ID 075018, 2013.

[67] Z. Zheng, X. Sun, and Y. Lei, "Monitoring corrosion of reinforcement in concrete structures via fiber Bragg grating sensors," Frontiers of Mechanical Engineering in China, vol. 4, no. 3, pp. 316-319, 2009.

[68] Z. Zheng, Y. Lei, and X. Sun, "Measuring corrosion of steels in concrete via fiber Bragg grating sensors-lab experimental test and in-field application," in Proceedings of the Earth and Space Conference, pp. 2422-2430, Honolulu, Hawaii, USA, March 2010.

[69] J.-P. Cai and S. B. Lyon, "A mechanistic study of initial atmospheric corrosion kinetics using electrical resistance sensors," Corrosion Science, vol. 47, no. 12, pp. 2956-2973, 2005.

[70] A. A. Sagüés and M. T. Walsh, "Kelvin Probe electrode for contactless potential measurement on concrete-properties and corrosion profiling application," Corrosion Science, vol. 56, pp. 26-35, 2012.

[71] A. Legat, M. Leban, and Ž. Bajt, "Corrosion processes of steel in concrete characterized by means of electrochemical noise," Electrochimica Acta, vol. 49, no. 17-18, pp. 2741-2751, 2004.

[72] A. Česen, T. Kosec, and A. Legat, "Characterization of steel corrosion in mortar by various electrochemical and physical techniques," Corrosion Science, vol. 75, pp. 47-57, 2013.

[73] A. Michel, B. J. Pease, M. R. Geiker, H. Stang, and J. F. Olesen, "Monitoring reinforcement corrosion and corrosion-induced cracking using non-destructive X-ray attenuation measurements," Cement and Concrete Research, vol. 41, no. 11, pp. 10851094, 2011.

[74] B. Ingham, M. Ko, N. Laycock et al., "In situ synchrotron Xray diffraction study of scale formation during $\mathrm{CO}_{2}$ corrosion of carbon steel in sodium and magnesium chloride solutions," Corrosion Science, vol. 56, pp. 96-104, 2012.

[75] J. Wei, X. X. Fu, J. H. Dong, and W. Ke, "Corrosion evolution of reinforcing steel in concrete under dry/wet cyclic conditions contaminated with chloride," Journal of Materials Science \& Technology, vol. 28, no. 10, pp. 905-912, 2012.

[76] M. Outirite, M. Lagrenée, M. Lebrini et al., "Ac impedance, Xray photoelectron spectroscopy and density functional theory studies of 3,5-bis(n-pyridyl)-1,2,4-oxadiazoles as efficient corrosion inhibitors for carbon steel surface in hydrochloric acid solution," Electrochimica Acta, vol. 55, no. 5, pp. 1670-1681, 2010.

[77] B. Fernandes, M. Titus, D. K. Nims, A. Ghorbanpoor, and V. K. Devabhaktunia, "Practical assessment of magnetic methods for corrosion detection in an adjacent precast, prestressed concrete box-beam bridge," Nondestructive Testing and Evaluation, vol. 28, no. 2, pp. 99-118, 2012.
[78] Y. Dost, N. Apaydın, E. Dedeoğlu, D. K. MacKenzie, and O. Z. Akkol, "Non-destructive testing of Bosphorus bridges," in Nondestructive Testing of Materials and Structures, vol. 6 of RILEM Bookseries, pp. 819-825, 2013.

[79] L. Jones, S. Pessiki, C. Naito, and I. Hodgson, "Inspection methods \& techniques to determine non visible corrosion of prestressing strands in concrete bridge components task 2-assessment of candidate NDT methods," Transportation Research Board of the National Academies, 2010, http://trid.trb .org/.

[80] B. Fernandes, M. Titus, D. K. Nims, A. Ghorbanpoor, and V. Devabhaktuni, "Field test of magnetic methods for corrosion detection in prestressing strands in adjacent box-beam bridges," Journal of Bridge Engineering, vol. 17, no. 6, pp. 984-988, 2012.

[81] K. Yuma, W. Tomoyo, K. Tomoe, and O. Masayasu, "Corrosion mechanisms in reinforced concrete by acoustic emission," Construction and Building Materials, vol. 48, pp. 1240-1247, 2013.

[82] M. Ohtsu, K. Mori, and Y. Kawasaki, "Corrosion process and mechanisms of corrosion-induced cracks in reinforced concrete identified by AE analysis," Strain, vol. 47, no. 2, pp. 179-186, 2011.

[83] L. Calabrese, G. Campanella, and E. Proverbio, "Noise removal by cluster analysis after long time AE corrosion monitoring of steel reinforcement in concrete," Construction and Building Materials, vol. 34, pp. 362-371, 2012.

[84] L. Calabres, G. Campanella, and E. Proverbio, "Identification of corrosion mechanisms by univariate and multivariate statistical analysis during long term acoustic emission monitoring on a pre-stressed concrete beam," Corrosion Science, vol. 73, pp. 161171, 2013.

[85] H. A. Elfergani, R. Pullin, and K. M. Holford, "Damage assessment of corrosion in prestressed concrete by acoustic emission," Construction and Building Materials, vol. 40, pp. 925-933, 2013.

[86] M. di Benedetti, G. Loreto, F. Matta, and A. Nanni, "Acoustic emission monitoring of reinforced concrete under accelerated corrosion," Journal of Materials in Civil Engineering, vol. 25, no. 8, pp. 1022-1029, 2013. 


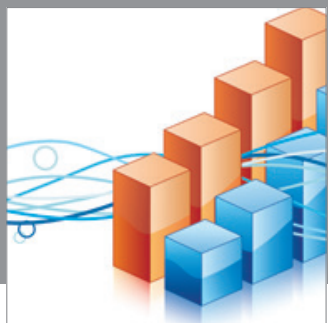

Advances in

Operations Research

mansans

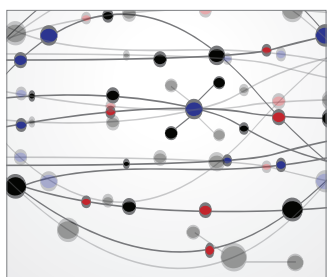

The Scientific World Journal
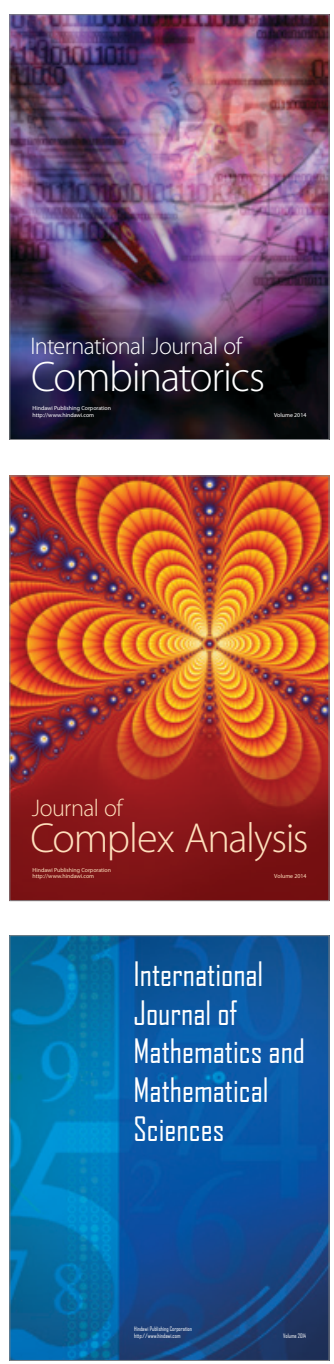
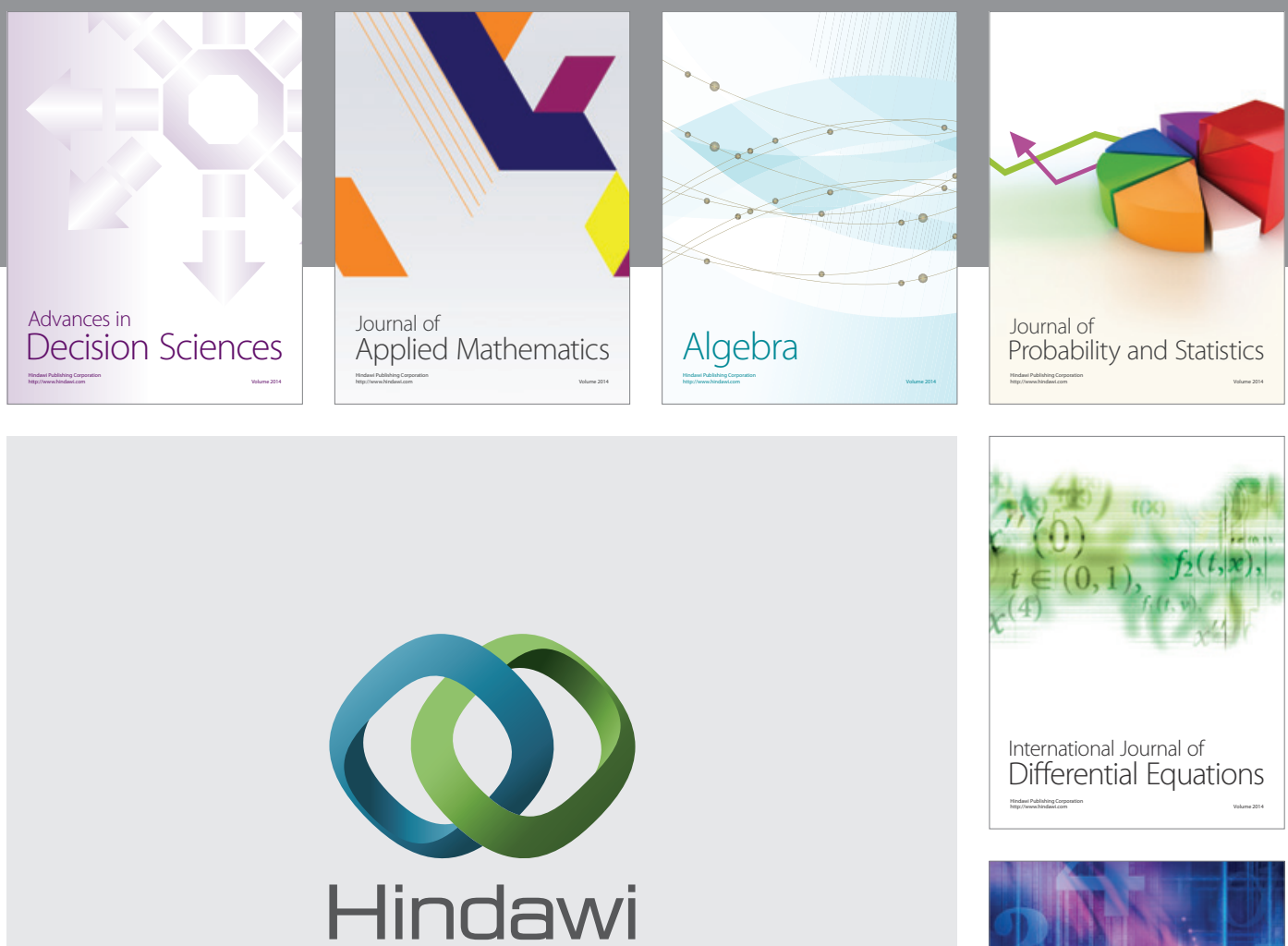

Submit your manuscripts at http://www.hindawi.com
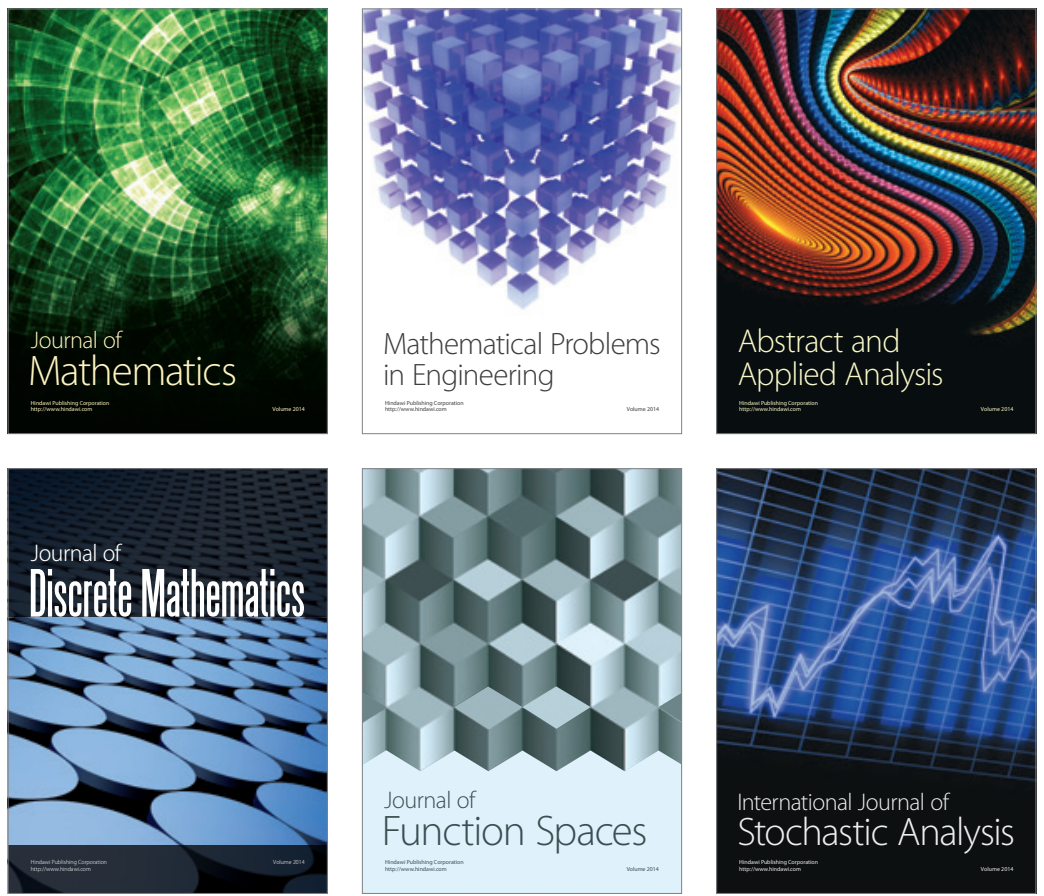

Journal of

Function Spaces

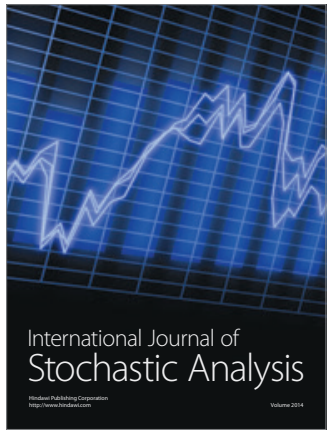

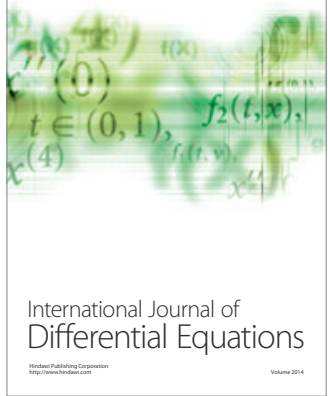
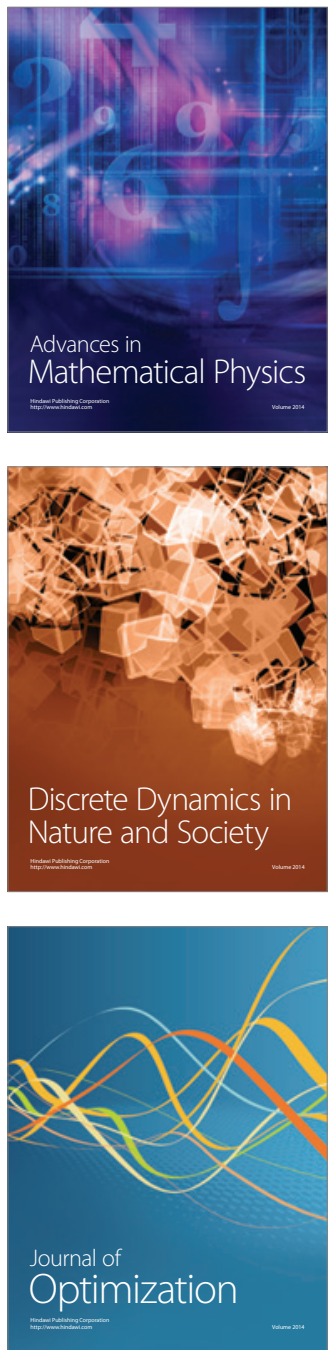